\title{
THE COMPLETION OF A THEOREM OF KANTOR
}

GERALD B. HUFF

The nature of the problem. Let a homaloidal net be defined by its order $n$ and its multiplicities $s_{1}, s_{2}, \cdots, s_{\rho}$ at set $P_{\rho}^{2}$ of $\rho$ general points in the plane. The positive integers $n, s_{1}, s_{2}, \cdots, s_{\rho}$ satisfy the equations

$$
\begin{aligned}
& s_{1}^{2}+s_{2}^{2}+\cdots+s_{\rho}^{2}-n^{2}=-1, \\
& s_{1}+s_{2}+\cdots+s_{\rho}-3 n=-3 .
\end{aligned}
$$

A planar Cremona transformation $C$ is set up by putting this net into projective correspondence with a net of lines in another plane. If a complete and regular linear system $\Sigma_{p, d}$ of dimension $d$, the generic curve of the system having the genus $p$, has the order $x_{0}$ and the multiplicities $x_{1}, x_{2}, \cdots, x_{\rho}$, then $x=\left\{x_{0} ; x_{1}, \cdots, x_{\rho}\right\}$ is called the characteristic of $\Sigma_{p, d}$. The image of $\Sigma_{p, d}$ under $C$ is another linear system of the same $p, d$ and a characteristic $x^{\prime}=\left\{x_{0}^{\prime} ; x_{1}^{\prime}, \cdots, x_{\rho}^{\prime}\right\}$ at the set $Q_{\rho}^{2}$ of the fundamental points of $C^{-1} . x^{\prime}$ is related to $x$ by the substitution

$$
\begin{aligned}
& x_{0}^{\prime}=n x_{0}-r_{1} x_{1}-\cdots-r_{\rho} x_{\rho}, \\
& x_{1}^{\prime}=s_{1} x_{0}-\alpha_{11} x_{1}-\cdots-\alpha_{1 \rho} x_{\rho} \text {, } \\
& x_{\rho}^{\prime}=s_{\rho} x_{0}-\alpha_{\rho 1} x_{1}-\cdots-\alpha_{\rho \rho} x_{\rho} .
\end{aligned}
$$

The sets of numbers $\left\{s_{i}, \alpha_{i 1}, \cdots, \alpha_{i \rho}\right\}$ are the characteristics of the principal curves of $C$ at $P_{\rho}^{2}$ and satisfy the equations

$$
\begin{aligned}
& \alpha_{i 1}^{2}+\alpha_{i 2}^{2}+\cdots+\alpha_{i \rho}^{2}-s_{i}^{2}=1, \\
& \alpha_{i 1}+\alpha_{i 2}+\cdots+\alpha_{i \rho}-3 s_{i}=-1 .
\end{aligned}
$$

The linear substitution (2) has as absolute invariants the forms

$$
\begin{aligned}
(x x) & \equiv x_{1}^{2}+x_{2}^{2}+\cdots+x_{\rho}^{2}-x_{0}^{2} \\
(l x) & \equiv x_{1}+x_{2}+\cdots+x_{\rho}-3 x_{0} .
\end{aligned}
$$

The problems considered in this paper arise from the fact that the converses of two of the above statements do not hold. There are sets of positive integers satisfying equations (1) which are not associated with any homaloidal net and there are linear substitutions (2) which

Presented to the Society, April 29, 1944; received by the editors April 17, 1944. 
leave $(x x),(l x)$ invariant which are not associated with any planar Cremona transformation. A solution of (1) or a linear substitution (2) which does have the geometric meaning described above will be said to be proper.

In his prize memoir [8] ${ }^{1}$ of $1884, S$. Kantor stated the following proposition: a linear substitution (2) leaving $(x x)$ and $(l x)$ invariant and in which $n, s_{i}, r_{j}, \alpha_{i j}$ are non-negative integers is proper. He gave two "proofs" of the assertion. In 1931, Cooolidge [4] recognized the importance of the proposition and supported it with an argument like one of Kantor's. At the time Coble [1] pointed out that the proof was not valid. In 1934, this writer [5] constructed an example $(\rho=11)$ which showed that the proposition was not true; and, in 1940, by using the specific results [2] of Coble on irreducible solutions of (3), it was possible to prove $[6$, p. 865] that Kantor's theorem was true for $\rho<11$.

The purpose of this paper is to exhibit further necessary conditions on a proper linear substitution which will also be sufficient for all values of $\rho$.

\section{Two lemmas.}

Lemma 1. Let $\{p\}=\left\{p_{0} ; p_{1}, \cdots, p_{p}\right\}$ be an integer solution of equations (3) such that $p_{0} \geqq 0$ and $p_{1} \geqq p_{2} \geqq \cdots \geqq p_{p}$. Then $2 p_{0}-p_{1}-p_{2}-p_{3}$ $\geqq 0$.

It is easily verified that for each of $p_{0}=0,1$ there is a unique solution, and that each satisfies the lemma. In the case $p_{0}>1$, it may be shown that $p_{0}>p_{1}$. Indeed, $p_{0}=p_{1}$ requires that $p_{0}=0,1$ and it is easy to show that $p_{1}>p_{0}$ is impossible for $p_{0}>1$. Thus for $p_{0}>1$ we may write

$$
p_{1}=p_{0}-b_{1} ; \quad p_{2}=p_{0}-b_{2} ; \quad p_{3}=p_{0}-b_{3},
$$

where $0<b_{1} \leqq b_{2} \leqq b_{3}$. Substitution in the quadratic relation of (3) yields

$$
p_{4}^{2}+\cdots+p_{p}^{2}+b_{1}^{2}+b_{2}^{2}+b_{3}^{2}+2 p_{0}^{2}=1+2\left(b_{1}+b_{2}+b_{3}\right) p_{0} .
$$

But

$$
3 \leqq b_{1}^{2}+b_{2}^{2}+b_{3}^{2}+p_{4}^{2}+\cdots+p_{\rho}^{2} .
$$

Adding these yields

$$
2\left(p_{0}^{2}+1\right) \leqq 2\left(b_{1}+b_{2}+b_{3}\right) p_{0} .
$$

${ }^{1}$ Numbers in brackets refer to the bibliography at the end of the paper. 
Hence,

or

$$
\begin{gathered}
p_{0}<b_{1}+b_{2}+b_{3}, \\
3 p_{0}-b_{1}-b_{2}-b_{3}<2 p_{0} ;
\end{gathered}
$$

$$
p_{1}+p_{2}+p_{3}<2 p_{0}
$$

The restriction that $p_{1} \geqq p_{2} \geqq \cdots \cdots p_{\rho}$ is not essential to the proof. Indeed, it is clear that if the inequality holds for the three largest of $p_{i}$, then it must hold for any three.

LEMMA 2. If an integer linear substitution $L(I)$ of the form (2) leaves $(x x),(l x)$ invariant, and if the numbers $\left\{n ; r_{j}\right\}$ constitute a proper solution of (1), then the $L(I)$ is proper.

Since the characteristic $\left\{n ; r_{j}\right\}$ is proper, it is associated with a Cremona transformation which has the description

$$
\begin{aligned}
& x_{0}^{\prime}=n x_{0}-\sigma_{1} x_{1}-\cdots-\sigma_{\rho} x_{\rho}, \\
& x_{1}^{\prime}=r_{1} x_{1}-\beta_{11} x_{1}-\cdots-\beta_{1 \rho} x_{\rho}, \\
& \cdot \cdot \cdot \cdot \cdot \cdot \cdot \cdot \cdot \cdot \cdot \cdot, \\
& x_{\rho}^{\prime}=r_{\rho} x_{1}-\beta_{\rho 1} x_{1}-\cdots-\beta_{\rho \rho} x_{\rho} .
\end{aligned}
$$

Consider the product ${ }^{2} L(I) L(C)$ as being in the form (2) with primed coefficients. It is a linear substitution with $(x x)$ and $(l x)$ as invariants and hence $[3]$ :

(i) $\left\{n^{\prime} ; s_{i}^{\prime}\right\}$ and $\left\{n^{\prime} ; r_{j}^{\prime}\right\}$ satisfy (1);

(ii) $\left\{s_{i}^{\prime} ; \alpha_{i j}^{\prime}\right\}$ and $\left\{r_{j}^{\prime} ; \alpha_{i j}^{\prime}\right\}$ satisfy (3); and

(iii) $s_{i}^{\prime} s_{k}^{\prime}-\alpha_{i 1}^{\prime} \alpha_{k 1}^{\prime}-\alpha_{i 2}^{\prime} \alpha_{k 2}^{\prime}-\cdots-\alpha_{i \rho}^{\prime} \alpha_{k \rho}^{\prime}=0, i \neq k$.

Now $n^{\prime}=n^{2}-r_{1}^{2}-\cdots-r_{\rho}^{2}=1$, and hence, from (i), $r_{1}^{\prime}=\cdots=r_{\rho}^{\prime}$ $=s_{1}^{\prime}=\cdots=s_{\rho}^{\prime}=0$. Since the characteristics $\left\{s_{i}^{\prime} ; \alpha_{i j}^{\prime}\right\}\left[r_{j}^{\prime} ; \alpha_{i j}^{\prime}\right]$ satisfy (3), have $s_{i}^{\prime}=0\left[r_{j}^{\prime}=0\right]$ and are integral, they must be of type $\left\{0 ; 0^{p-1}-1\right\}$. From (iii) it is clear that if $\alpha_{i j}^{\prime}=-1$, and $\alpha_{k} i=-1$, then $j \neq l$. $L(I) L(C)$ must then be the identity or a permutation $P$ of the letters $x_{1}, \cdots, x_{\rho}$. Then $L(I)=L^{-1}(C)$ or $L(I)=P L^{-1}(C)$. In either case, $L(I)$ is proper.

\section{The proof of the theorem.}

Theorem 1. A linear substitution $L$ of the form (2) is proper if and only if:

(a) the coefficients are integers,

(b) $(x x)$ and $(l x)$ are absolute invariants, and

2 The convention for order of multiplication is $L(C)$ followed by $L(I)$. 
(c) $p_{0}^{\prime}$ has the same sign as $p_{0}$ if $\{p\}$ is a solution of (3) and $L\{p\}=\left\{p^{\prime}\right\}$.

The necessity of conditions (a), (b) is well known [9]. Now a geometric $L(C)$ may be expressed as the product of linear substitutions which are permutations of $x_{1}, \cdots, x_{\rho}$, and of

$A_{128}:$

$$
x_{0}^{\prime}=2 x_{0}-x_{1}-x_{2}-x_{3},
$$$$
x_{i}^{\prime}=x_{i}+\left(x_{0}-x_{1}-x_{2}-x_{3}\right), \quad i=1,2,3,
$$$$
x_{i}^{\prime}=x_{j} \text {, }
$$$$
j=4, \cdots, \rho \text {. }
$$

By Lemma 1 it is clear that under $A_{123}$ any solution $\{p\}$ of (3) with $p_{0} \geqq 0$ goes into a $\left\{p^{\prime}\right\}$ of $p_{0}^{\prime} \geqq 0$. Since $A_{123}$ is an involution, it must then send a $\{p\}$ of $p_{0}<0$ into a $\left\{p^{\prime}\right\}$ of $p_{0}^{\prime}<0 . L(C)$ must then have the same property.

The proof of the sufficiency of the conditions depends on the following theorem:

THEOREM 2 [7]. Let $\{\gamma\}$ be an integer solution of equations (1) arranged so that $\gamma_{1} \geqq \gamma_{2} \geqq \cdots \geqq \gamma_{\rho}$. Further, let $\{\gamma\}$ satisfy the finite set of inequalities $p_{0} \gamma_{0}-p_{1} \gamma_{1}-\cdots-p_{\rho} \gamma_{\rho} \geqq 0$, where the characteristics $\{p\}$ run over the finite set of all proper solutions of (3) with $p_{0}<\gamma_{0}$ and so ordered that $p_{1} \geqq p_{2} \geqq \cdots \geqq p_{\rho}$. Then $\{\gamma\}$ is the characteristic of a homaloidal net.

The ordering of $\{\gamma\}$ and $\{p\}$ is not necessary, but is stated for emphasis.

To demonstrate that an $L$ of the form (2) satisfying (a), (b), (c) is proper, note first that $\left\{n ; r_{1}, \cdots, r_{p}\right\}$ is an integer solution of (1) as a consequence of (a), (b), and (i). The invariance of the sign of $p_{0}$ assures that the inequalities of Theorem 2 hold. Thus $\left\{n ; r_{1}, \cdots, r_{p}\right\}$ is the characteristic of a homaloidal net. By Lemma 2, the linear substitution $L$ is proper.

The restriction of integer coefficients is indeed essential. An example has been exhibited [6, p. 863] for $\rho=9$ which satisfies all other conditions and is not an $L(C)$ since the numbers $s_{i}, \alpha_{i j}$ are rational. The proof of the sufficiency of the conditions of Theorem 1 could be proved by the method Coolidge uses; under the conditions given here, that argument is valid.

\section{Some results.}

Corollary 1. If a solution $\{p\}$ of equations (3) satisfies

$$
p_{0} c_{0}-p_{1} c_{1}-\cdots-p_{\rho} c_{\rho} \geqq 0
$$


for a single proper $\{c\}$ which is a solution of (1), then it satisfies that relation for every proper $\{c\}$.

Corollary 2. Given solutions $\{c\},\{p\}$ of (1) and (3) such that $p_{0}>0, p_{0} c_{0}-p_{1} c_{1}-\cdots-p_{p} c_{0}<0$, then $\{c\}$ is not proper.

Corollary 3. The linear substitution group generated by $A_{123}$ and the permutations of $x_{1}, x_{2}, \cdots, x_{\rho}$ is completely characterized by conditions (a), (b), (c) of Theorem 1.

The above results follow easily from Theorem 1. A similar theorem for characteristics for which $x_{0}$ never vanishes would be useful.

\section{BIBLIOGRAPHY}

1. A. B. Coble, Amer. Math. Monthly vol. 39 (1932) p. 293.

2. - Cremona's diophantine equations, Amer. J. Math. vol. 56 (1934) p. 459.

3. - Algebraic geometry and theta functions, Amer. Math. Soc. Colloquium Publications, vol. 10, New York, 1929, p. 11.

4. J. L. Coolidge, Algebraic plane curves, Oxford, 1931, p. 487.

5. G. B. Huff, A note on Cremona transformations, Proc. Nat. Acad. Sci. U.S.A. vol. 20 (1934) p. 428.

6. - A geometry associated with Cremona's equations, Amer. J. Math. vol. 62 (1940) pp. 865 and 863.

7. - A sufficient condition that a C-characteristic be geometric, Proc. Nat. Acad. Sci. U.S.A. vol. 29 (1943) p. 198.

8. S. Kantor, Premiers fondements pour une theorie des transformations univoques, Naples, 1891, p. 297.

9. National Research Council Report, Selected topics in algebraic geometry, Bulletin No. 63, Washington, D. C., 1929, p. 86.

SOUTHERN METHODIST UNIVERSITY 